\title{
Low frequency geomagnetic field variations at Dome C (Antarctica)
}

\author{
S. Lepidi ${ }^{1}$, L. Cafarella ${ }^{2}$, P. Francia ${ }^{3}$, A. Meloni ${ }^{2}$, P. Palangio ${ }^{1}$, and J. J. Schott ${ }^{4}$ \\ ${ }^{1}$ Istituto Nazionale di Geofisica e Vulcanologia, L'Aquila, Italy \\ ${ }^{2}$ Istituto Nazionale di Geofisica e Vulcanologia, Roma, Italy \\ ${ }^{3}$ Dipartimento di Fisica, Università dell' Aquila, L'Aquila, Italy \\ ${ }^{4}$ EOST, IPG, Strasbourg, France
}

Received: 17 January 2002 - Revised: 7 June 2002 - Accepted: 28 October 2002

\begin{abstract}
We conduct an analysis of the geomagnetic field variations recorded at the new Antarctic station Dome C, located very close to the geomagnetic pole, which has been operating for approximately one month during the 1999-2000 campaign. We also perform a comparison with simultaneous measurements at the Italian Antarctic station Terra Nova Bay, in order to investigate the spatial extension of the phenomena observed at very high latitude. Our results show that between the two stations the daily variation is similar and the fluctuations with $\mathrm{f} \sim 1 \mathrm{mHz}$ are coherent, provided that in both cases the comparison is made between geographically oriented components, suggesting that ionospheric currents related to the geographic position, more than field-aligned currents, are responsible for the lowest frequency variations; conversely, higher frequency (Pc5) fluctuations are substantially decoupled between the two stations. We also found that at Dome C the fluctuation power in the $0.55-6.7 \mathrm{mHz}$ frequency band is well related with the solar wind speed during the whole day and that at Terra Nova Bay the correlation is also high, except around local geomagnetic noon, when the station approaches the polar cusp. These results indicate that the solar wind speed control of the geomagnetic field fluctuation power is very strict in the polar cap and less important close to the polar cusp.
\end{abstract}

Key words. Magnetospheric physics (MHD waves and instabilities; Polar cap phenomena; Solar wind-magnetosphere interactions)

\section{Introduction}

The study of the geomagnetic field variations in Antarctica is important in that local field lines are close to the extreme magnetospheric regions (for instance the magnetopause and the polar cusp) where several generation mechanisms for ULF waves are active (Arnoldy et al., 1988 and references therein). In this sense, ground measurements at the Antarc-

Correspondence to: S. Lepidi (lepidi@ingv.it) tic stations have provided useful information on the local and/or temporal extent of the Pc5 $(1.6-6.7 \mathrm{mHz})$ and lower frequency pulsations (the so called IPCL, $\sim 1-5 \mathrm{mHz}$; Troitskaya et al., 1973) and on their relationship with solar wind (SW) parameters. In particular, enhancements of the wave activity were clearly observed around local magnetic noon (Olson, 1986; Kleimenova et al., 1989; Engebretson et al., 1995; Ballatore et al., 1996; Kurazhkovskaya and Klain, 2000), i.e. when the stations are expected to approach the cusp position, while the presence of a power enhancement near magnetic midnight was explained in terms of substorm related features (Olson, 1986). The power of the Pc5 pulsations was found to be strongly dependent on the SW speed (Wolfe et al., 1987; Engebretson et al., 1995, 1998; Ballatore et al., 1996), indicating a wave source related to the KelvinHelmholtz instability on the flanks of the magnetopause. Conversely, no clear relationship with the north-south component of the interplanetary magnetic field has been observed (Engebretson et al., 1995; Ballatore et al., 1998). Ballatore et al. (1998) have also shown that the Pc5 pulsation power at $\sim 80 \mathrm{~S}$ corrected geomagnetic latitude does not show a strong dependence on the geomagnetic activity.

The availability of data from approximately 1 month from a new Antarctic station at Concordia, in the framework of a joint French-Italian venture at Dome C (DMC), located very close to the geomagnetic pole, allows us to extend the study of the geomagnetic variations at the footprint of open field lines stretching in the geomagnetic tail.

For a comparison, we also analyzed the simultaneous data recorded at the Italian Antarctic station Terra Nova Bay (TNB). The location of the two stations is particularly interesting in that they are approximately at the same geographic latitude but at quite different corrected geomagnetic latitude. Indeed, TNB during the major part of the day is situated in the polar cap but around geomagnetic noon approaches the polar cusp and, in particular magnetospheric conditions, can even reach the location of closed field lines; conversely DMC is located deep in the polar cap during the whole day.

TNB has operated since $1986-87$ as an observatory and 

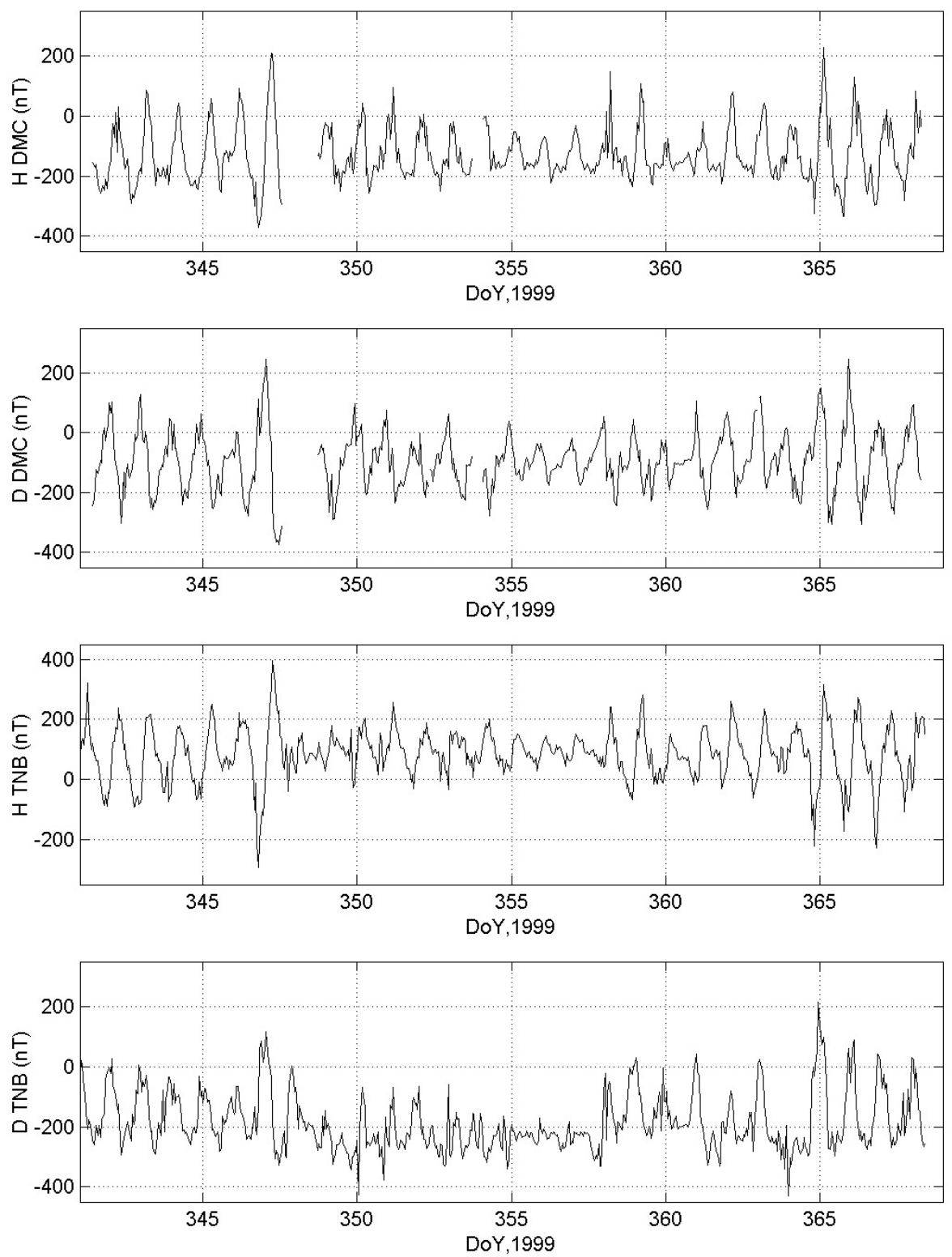

Fig. 1. Hourly averages of the $H$ and $D$ components at DMC and TNB from 7 December 1999 to 3 January 2000. Times are in UT.

more recently also with high resolution $(1 \mathrm{~s})$ measurements. The availability of long series of data allowed us to conduct several studies to characterize the diurnal variation $(\mathrm{Ca}$ farella et al., 1998), as well as the local geomagnetic field fluctuations and their relationship with the SW parameters (for a review on ULF fluctuations at TNB see Villante et al., 2000a). For the purpose of the present investigation, it is interesting to recall some of the results obtained for the lower frequency variations. In particular, the statistical studies by Ballatore et al. (1996) and Villante et al. (2000b) have shown that the power level of low frequency pulsations (Pc3-Pc5) maximizes around local geomagnetic noon, when TNB station approaches the polar cusp; moreover there is a good correlation between the fluctuation power and the SW speed, indicating a wave source related to the Kelvin-Helmholtz in- stability. From a statistical analysis, Villante et al. (1997) also found evidence, in the average daytime spectra of the $H$ component, for power enhancements at discrete frequencies of the order of few $\mathrm{mHz}$. This was more evident during time intervals characterized by high SW speed. The observed frequencies are close to those detected at auroral latitudes in the F-region drift velocities and in the geomagnetic field fluctuations and interpreted in terms of field line resonances excited by magnetospheric cavity/waveguide modes (Samson et al., 1992; Walker et al., 1992; Ziesolleck and McDiarmid, 1994, 1995). Due to external stimulation, for example SW pressure pulses or Kelvin-Helmholtz instability, compressional waves are generated inside the magnetosphere and propagate tailward reflecting between two boundaries, such as the magnetopause and the plasmapause, giving rise to standing waves 

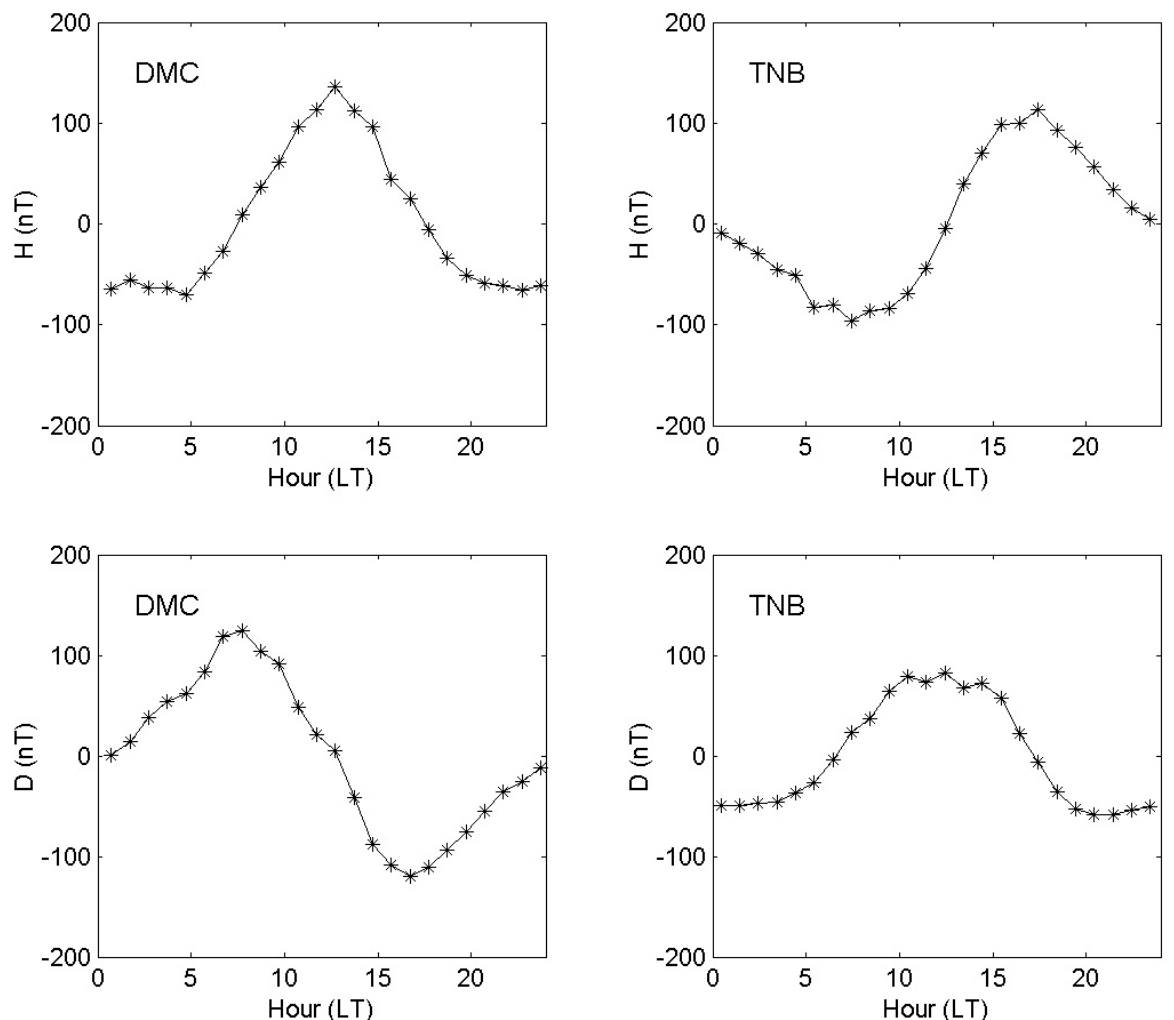

Fig. 2. Average daily variation of the $H$ and $D$ hourly values at DMC and TNB.

at discrete frequencies. The evidence of the power enhancements at TNB, clearer near local geomagnetic noon, suggests that the fluctuations can extend some finite distance into the polar cap (see also Matthews et al., 1996).

In this paper we extend the analysis of the geomagnetic field low frequency variations up to the latitude of the geomagnetic pole and, comparing simultaneous measurements at TNB and DMC, address the question of the spatial extension of the phenomena observed at very high latitude.

\section{Data analysis and experimental observations}

The station DMC was installed during the 1999-2000 expedition, when instruments and shelter were tested in the planning phases of a permanent observatory and, after some tests and checks, the observatory started in operation on 7 December 1999 and worked until 3 January 2000. The variations of the geomagnetic field components were measured by means of a FGE DMI variometer with a cardanic suspension, magnetically oriented, and the acquisition was triggered by a GPS.

We analyzed the 1 min values of the horizontal $H$ and $D$ geomagnetic field components measured at the two Antarctic stations DMC and TNB by a fluxgate magnetometer in the time period from 7 December 1999 to 3 January 2000. In Table 1 the geographic coordinates, the IGRF99 corrected geomagnetic coordinates and the time in UT of the magnetic
Table 1. Geographic coordinates, IGRF99 corrected geomagnetic coordinates and time in UT of the magnetic local midnight for the stations DMC and TNB

\begin{tabular}{cccc}
\hline Station & Geographic Coord. & Corr. Geom. Coord. & MLTMN (UT) \\
\hline DMC & 75.11 S 123.40E & 88.83 S 55.62 E & $1: 04$ \\
TNB & 74.69 S 164.12E & 80.03 S 307.74 E & $8: 06$ \\
\hline
\end{tabular}

local midnight for the two stations are reported. Magnetic local time (MLT), expecially at high latitudes, is an important parameter to understand several physical phenomena. However, MLT becomes meaningless approaching the geomagnetic pole, where it is not defined. For this reason, in the following we use the geographic local time (LT) as an ordering parameter of the observed phenomena, indicating also the MLT only for TNB station.

\subsection{Diurnal variation}

In Fig. 1 we show the hourly averages of the $H$ and $D$ components at the two stations; it is evident that a quite regular 24-h variation is present at both stations for both components. In order to better investigate this feature, we calculated the average daily variation of the amplitude of the horizontal components, after eliminating the average values of the time series (Fig. 2). As can be seen, at DMC the $H$ 

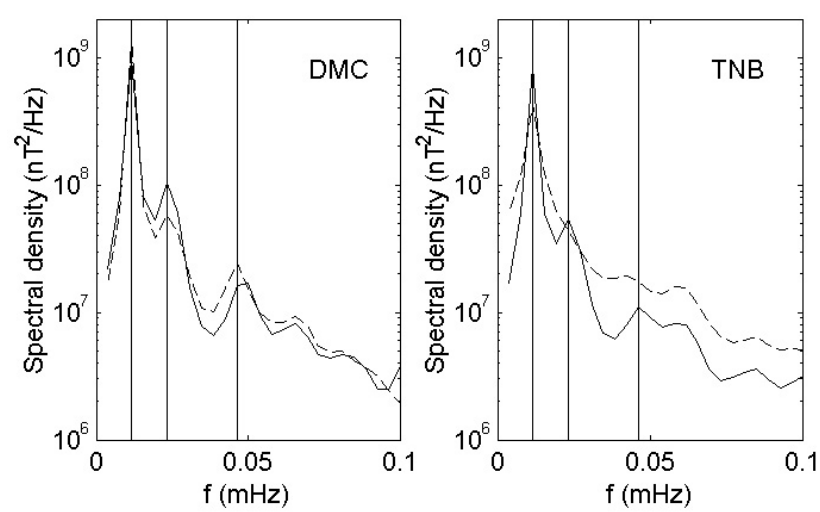

Fig. 3. Average power spectra of the $H$ and $D$ components (indicated with solid and dashed lines, respectively) at DMC and TNB computed from hourly values. Vertical lines indicate the frequencies corresponding to $24 \mathrm{~h}, 12 \mathrm{~h}$ and $6 \mathrm{~h}$.

component amplitude shows a symmetric variation with a maximum around local noon, while the $D$ component variation is antisymmetric around $\sim 12: 00 \mathrm{LT}$, with a maximum at $\sim 08: 00 \mathrm{LT}$ and a minimum at $\sim 16: 00 \mathrm{LT}$. On the contrary, at TNB a symmetric variation with a wide maximum around local noon characterizes the $D$ component, and an antisymmetric variation with a minimum around 08:00 LT and a maximum around 16:00 LT characterizes the $H$ component. The amplitude of the diurnal variation at the two stations is comparable: at DMC it is $\sim 200 \mathrm{nT}$ for $H$ and $\sim 250 \mathrm{nT}$ for $D$ and at TNB it is $\sim 200 \mathrm{nT}$ for $H$ and $\sim 150 \mathrm{nT}$ for $D$.

The clear diurnal variation is also evidenced by a spectral analysis of the hourly averages. We computed individual power spectra from 72-h values with a step size of $24 \mathrm{~h}$, obtaining 12 spectra for DMC (compatibly with some data gaps) and 25 spectra for TNB; the resulting average power spectra (Fig. 3) show a clear peak at $24 \mathrm{~h}$, corresponding to the diurnal variation, and, mainly at DMC, also evidence for minor peaks at $12 \mathrm{~h}$ and $6 \mathrm{~h}$, corresponding to its harmonics (Campbell, 1997). From a visual inspection of Fig. 1, it is evident that the amplitude of the variation varies from day to day, and we found that it closely follows the level of the magnetospheric activity. Indeed, as shown in Fig. 4, the logarithmic total horizontal power ( $H$ power $+D$ power) at 24 -h is well related to the corresponding average $K_{p}$ value, with a correlation coefficient of 0.89 and 0.84 for DMC and TNB, respectively (similar results are obtained separately for the two components).

\subsection{Low frequency fluctuations}

We next investigated the fluctuations in the frequency range $f=0.55-6.7 \mathrm{mHz}$ (i.e. $T=2.5-30 \mathrm{~min}$ ), approximately corresponding to the lower frequency pulsations, calculating the hourly power spectra of the $H$ and $D$ components from the original 1-min measurements. We found (Fig. 5) that the average power spectra at both stations are well approximated by a straight line on a log-log scale, and no clear power en-
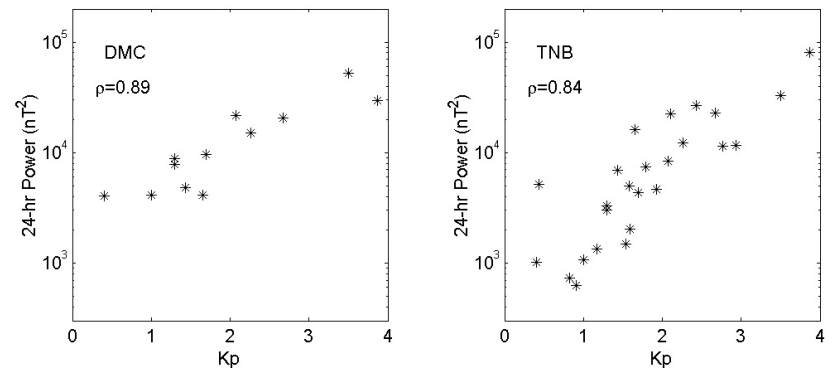

Fig. 4. The total $(H+D)$ amplitude of the 24 -h power peak at DMC and TNB versus the average $K_{p}$ index. The values of the correlation coefficients are also indicated.

hancement emerges in the analyzed frequency range; moreover, at DMC the $H$ and $D$ power levels are very similar, while at TNB a higher power content is present on the $D$ component. Comparing the two stations, it is also evident that at TNB there is a higher power content at the lower frequencies, while at DMC the power level is higher at the higher frequencies; this feature is well expressed by the spectral index (also indicated in Fig. 5), which is $\sim 3.0$ at TNB and $\sim 2.6$ at DMC for both components (we verified that the spectral index at both stations does not show a significant variation if we consider separately daytime and nighttime average spectra).

We studied the local time dependence of the low frequency fluctuation power (integrated over the $0.55-6.7 \mathrm{mHz}$ frequency range), logarithmically averaging the values corresponding to the same hour and plotting the behaviour in the $24 \mathrm{~h}$. It is evident (Fig. 6) that a different time behaviour emerges at the two stations: at DMC the most evident feature is that the low frequency power of the $H$ and $D$ components maximizes in the local early afternoon $(\sim 13: 00-15: 00 \mathrm{LT})$ and in the local late morning ( 09:00-11:00 LT), respectively; conversely at TNB both components show a very clear peak, with the largest power values on the $D$ component, at $\sim 07: 00 \mathrm{LT}$, corresponding to local geomagnetic noon. It is also interesting to note that during daytime hours the maximum power level detected at TNB is really higher than at DMC; conversely, during nighttime hours, when both TNB and DMC are situated deep in the polar cap, the power level at the two stations is very similar (it is even somewhat higher at DMC). Of course, the observed power values reflect primarily the power content of the lower frequency fluctuations; in order to evaluate whether the same local time dependence characterizes also the higher frequency fluctuations, we conducted the same analysis but considering only the frequency range $4.2-6.7 \mathrm{mHz}$. The results are very similar, although in this case, the power level at the two stations is comparable, for both the minimum and the maximum values.

In order to analyze the relationship between the low frequency fluctuations and the magnetospheric and SW conditions, we estimated the correlation coefficient $\rho$ between the logarithm of the daily average power and the daily average values of the $K_{p}$ index and the SW speed ( $V$, from ACE 

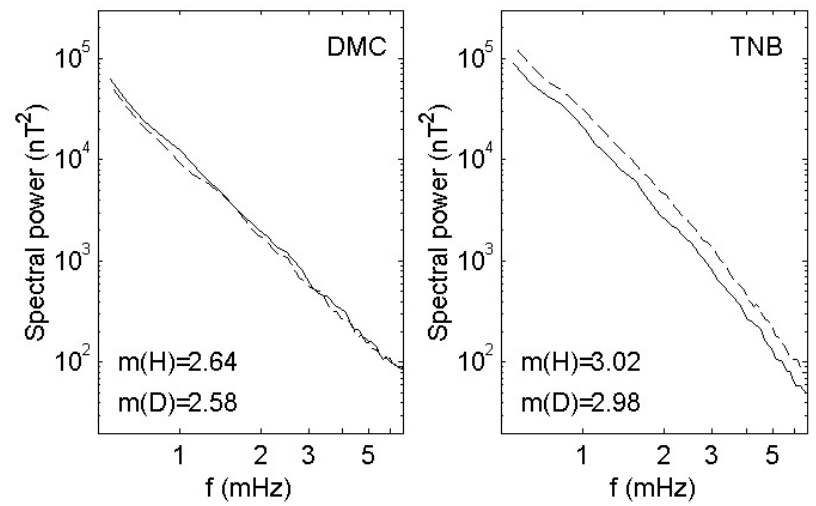

Fig. 5. The average power spectra of the $H$ and $D$ components (indicated with solid and dashed lines, respectively) at DMC and TNB computed from original 1-min values. The values of the spectral index $m$ are also indicated.

spacecraft) separately for the frequency bands $0.55-6.7 \mathrm{mHz}$ and $4.2-6.7 \mathrm{mHz}$. As can be seen from Table 2, the correlation coefficients with $K_{p}$ and $V$ are well above the $99.9 \%$ confidence limit $(\rho \sim 0.6)$, except for TNB in the frequency band $0.55-6.7 \mathrm{mHz}$. We also investigated the possible local time dependence of the correlation coefficient, considering hourly values of the low frequency power, $K_{p}$ and $V$ and, in order to have a higher statistical reliability, grouping together 3-h intervals starting from 0:00 UT. The results are shown in Fig. 7. As can be seen, at DMC the correlation with $K_{p}$ and $V$ is always well above the $99.9 \%$ confidence level (indicated in the figure by the dashed line) and does not show a clear local time dependence. With regards to TNB, the local time dependence is more clear, especially for the $D$ component, with a pronounced minimum of the correlation (in some cases with values lower than the $99.9 \%$ confidence level) in the local morning, when the power of the two components maximizes (Fig. 6). We also found (results not shown) that the low frequency power does not show any significant dependence on the interplanetary magnetic field orientation.

An analysis of the coherence $\gamma$ between the fluctuations at DMC and TNB (calculated over 30-min intervals and averaged over $3 \mathrm{~h}$ ) has revealed that for frequencies higher than $\sim 1.5 \mathrm{mHz}$ the fluctuations detected at the two stations are substantially decoupled (the average value of $\gamma$ is lower than 0.4 ). Conversely, for lower frequencies ( $f=0.55-1.1 \mathrm{mHz}$, i.e. $T=15-30 \mathrm{~min}$ ), the coherence can reach slightly higher values. Considering the homologous components of the geomagnetic field at the two stations, we found that the average value of $\gamma$ is higher than 0.5 between 09:00 and 15:00 UT for the $H$ component, and between 03:00 and 18:00 UT for the $D$ component (Fig. 8, upper panels). However, the occurrence of events with $\gamma>0.7$ (this threshold value allows us to select coherent events with a $95 \%$ confidence interval; Lepidi et al., 1999) is low, always lower than $40 \%$ during the day (Fig. 8, lower panels). The same analysis performed by cross-comparing the $H$ and $D$ components reveals that between non-homologous components the coher-
Table 2. Correlation coefficients between the logarithm of the daily average power at DMC and TNB and the daily average values of the $K_{p}$ index and the SW speed $V$, for the frequency bands 0.55 $6.7 \mathrm{mHz}$ and $4.2-6.7 \mathrm{mHz}$

\begin{tabular}{ccc}
\hline $\begin{array}{l}\text { Correlation } \\
\text { coefficient }\end{array}$ & $f=0.55-6.7 \mathrm{mHz}$ & $f=4.2-6.7 \mathrm{mHz}$ \\
\hline$H \mathrm{DMC}-K_{p}$ & 0.71 & 0.82 \\
$D \mathrm{DMC}-K_{p}$ & 0.79 & 0.82 \\
$H \mathrm{DMC}-V$ & 0.66 & 0.79 \\
$D \mathrm{DMC}-V$ & 0.75 & 0.84 \\
$H \mathrm{TNB}-K_{p}$ & 0.58 & 0.75 \\
$D \mathrm{TNB}-K_{p}$ & 0.59 & 0.82 \\
$H \mathrm{TNB}-V$ & 0.50 & 0.75 \\
$D \mathrm{TNB}-V$ & 0.57 & 0.81 \\
\hline
\end{tabular}

ence is higher (Fig. 9). Indeed, when considering the pair $D$ (DMC) - $H$ (TNB), in the time interval 09:00-12:00 UT the average coherence is $\sim 0.7$ and the occurrence of coherent events $(\gamma>0.7)$ is $\sim 50 \%$; the same results are obtained for the pair $H(\mathrm{DMC})-D(\mathrm{TNB})$ in the time interval 09:0015:00 UT. In all cases it is interesting to note that the most coherent low frequency fluctuations are statistically observed during time intervals characterized by a minor power content at both stations (Fig. 6).

\section{Summary and discussion}

In this paper we investigate the low frequency variations of the $H$ and $D$ geomagnetic field components measured at the two Antarctic stations DMC and TNB in the time period from 7 December 1999 to 3 January 2000, close to the maximum of solar activity. A comparison between DMC and TNB observations is particularly interesting in that the two stations are approximately situated at the same geographic latitude, but at different corrected geomagnetic latitude: DMC is located deep in the polar cap, very close to the geomagnetic pole, while TNB, which is also usually located in the polar cap, around local geomagnetic noon can reach and even cross the location of the polar cusp for particular magnetospheric conditions.

We first focused our attention on the daily variation of the geomagnetic field components and found that it appears to have a different local time behaviour at the two stations. However, when non-homologous components are compared, a close similarity of the daily variation at the two stations clearly emerges: the local time behaviour of $H$ (DMC) closely resembles that of $D$ (TNB) and the local time behaviour of $D(\mathrm{DMC})$ closely resembles, with opposite sign, that of $H(\mathrm{TNB})$. This result can be explained in terms of the electrical current systems which are responsible for the polar daily variation, including the poleward extension of the mid- and low-latitude ionospheric electric current vortices and the additional DP2 polar current system (Nishida and Kokubun, 1971). All these current systems, which are 

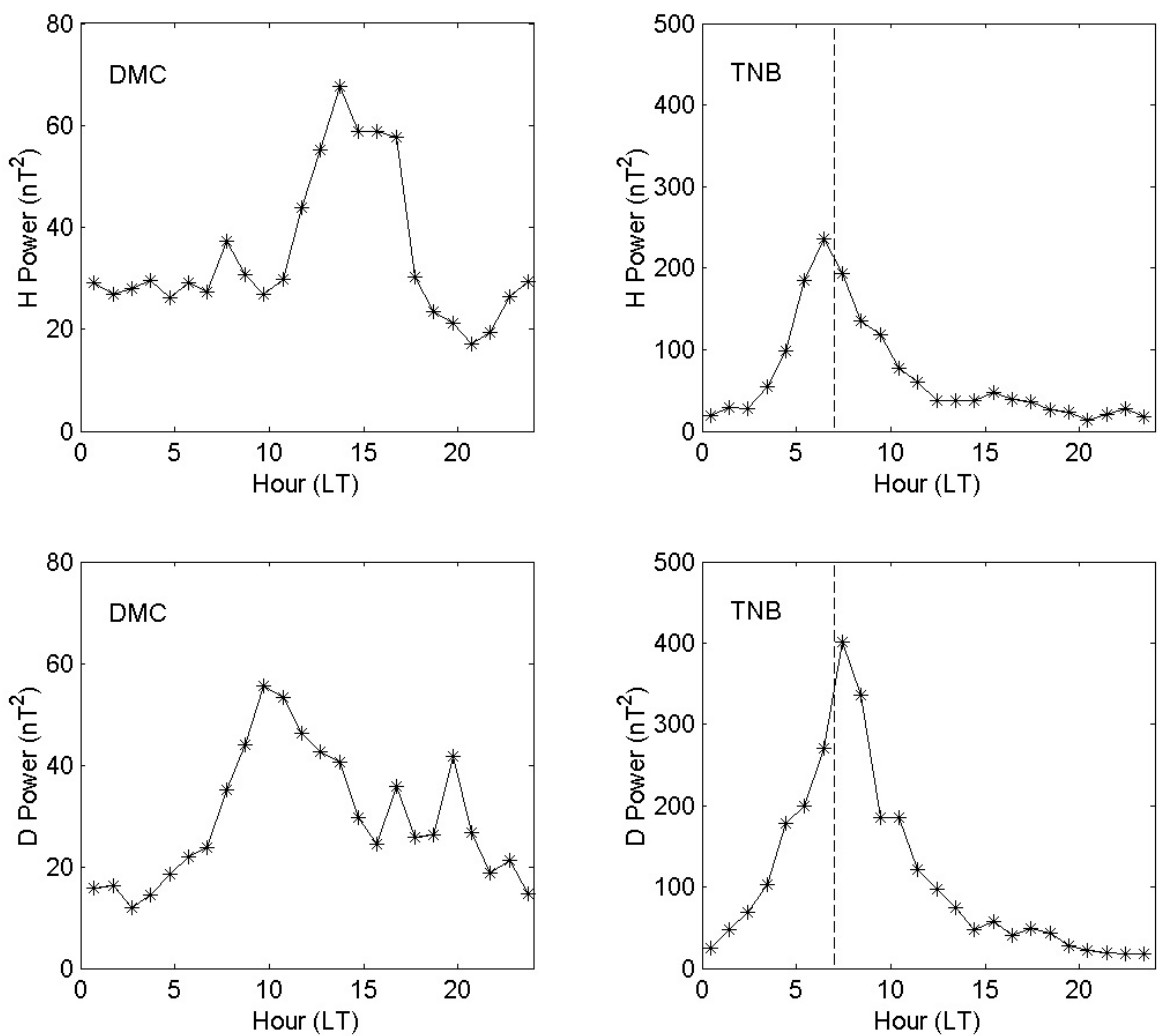

Fig. 6. Average local time dependence of the $0.55-6.7 \mathrm{mHz}$ fluctuation power at DMC and TNB. The vertical dashed lines indicate the local geomagnetic noon at TNB. Note the different vertical scales at the two stations.

stationary with respect to the Sun-Earth line, at stations located at the same geographic latitude give the same daily variation of the geomagnetic field along geographic directions (Campbell, 1989); since at TNB and DMC the instruments are magnetically oriented and the declination of the geomagnetic field at the two stations differs by $\sim 90^{\circ}$ (the IGRF99 declination values are $\sim 137^{\circ}$ at TNB and $\sim-137^{\circ}$ at DMC), similar daily variations are observed along perpendicular components. We note that the local time behaviour of the two horizontal components at DMC also closely resembles that observed on the homologous components at AGO P6 ( 85 S geomagnetic invariant latitude) by Detrick and Lanzerotti (2001) during geomagnetic quiet days, which, in turn, agrees well with the estimates of the $S q$ model by Campbell (1997). On this regard, we point out that the declination values at DMC and P6 are very similar $\left(\sim-137^{\circ}\right.$ and $-139^{\circ}$, respectively). As to the amplitude of the daily variation, at DMC and TNB it is roughly comparable, and is also similar to the one found at TNB during local summer and active years in a previous analysis by Cafarella et al. (1998).

A visual inspection of the geomagnetic data suggests that the daily variation is always present at both stations for both components (in this sense, the average power spectra show a pronounced power peak at $24 \mathrm{~h}$ and also minor peaks at $12 \mathrm{~h}$ and $6 \mathrm{~h}$ ), but its amplitude shows a large variation from day to day, being closely related to the $K_{p}$ index. This result indicates that in the polar region the strength of the cur- rent systems responsible for the daily variation is strongly dependent on the global level of the magnetospheric activity, and then shows a variability on time scales of days or less. A previous study of the daily variation at TNB, based on a long series of data, has provided evidence for a longer term modulation of its amplitude, due to a seasonal and solar cycle dependence (Palangio et al., 1996; Cafarella et al., 1998) and similar results have also been obtained at mid-latitudes (Campbell, 1989). We note that the daily variation at DMC and TNB is characterized by a greater excursion than the $S q$ variation at P6, reported by Detrick and Lanzerotti (2001). Since the $S q$ variation is calculated for the geomagnetic most quiet days, this difference can be well explained in terms of the observed dependence of the amplitude of the daily variation on magnetospheric conditions.

We also investigated the fluctuations in the frequency range $0.55-6.7 \mathrm{mHz}$, which correspond roughly to the lower frequency geomagnetic pulsations. The average power spectra show that at the lower frequencies the power content is higher at TNB, while at the higher frequencies it is higher at DMC. As to the spectral index, at TNB we found for both components a value of $\sim 3.0$, independent of local time, which is similar to that obtained in the Pc5 frequency band at approximately the same geomagnetic latitude (AGO P4, 80.0 S) during nighttime hours (Yagova et al., 2002) and also at slightly lower latitude (Iqaluit, $\sim 74.0 \mathrm{~N}$; De Lauretis et al., 1991). Conversely, Olson (1986) found that the spectral in- 

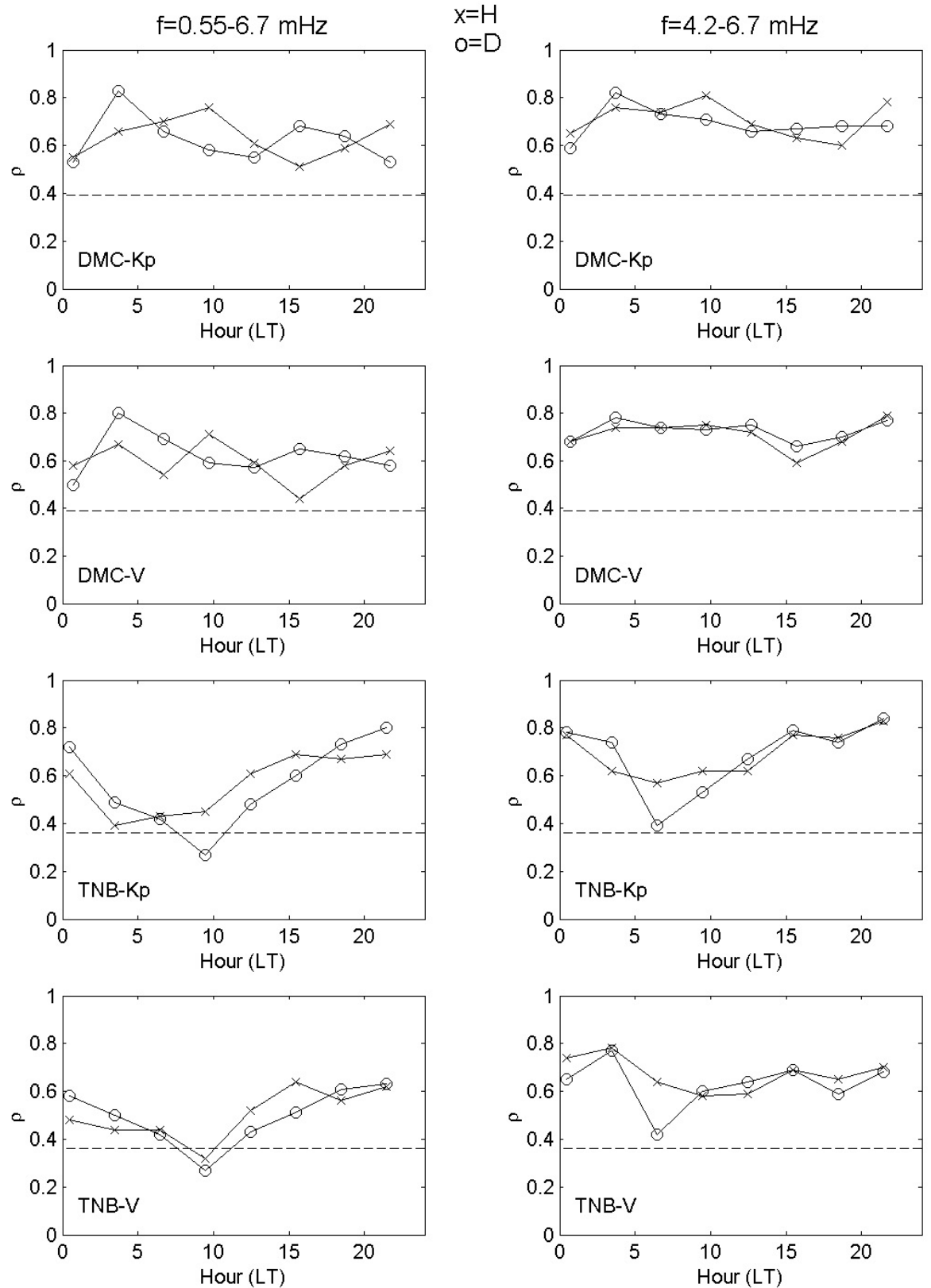

Fig. 7. Local time dependence of the correlation coefficient $\rho$ between the logarithm of the fluctuation power of the $H$ and $D$ components at the two stations and the $K_{p}$ index and the solar wind speed $V$, separately for the frequency bands $0.55-6.7 \mathrm{mHz}$ (left panels) and $4.2-6.7 \mathrm{mHz}$ (right panels). The horizontal dashed lines indicate the $\rho$ value corresponding to the $99.9 \%$ confidence level.

dex at Cape Perry $(73.8 \mathrm{~N})$ in the whole Pc1-Pc5 frequency range (i.e. in a frequency range really wider than that considered in the present analysis) is $\sim 2.6$. Our results also show that the spectral index at DMC ( 2.6 for both components) is slightly lower than at TNB, independent of the local time; on the contrary, Yagova et al. (2002) have shown that during nighttime hours the spectral index increases with increasing latitude inside the polar cap (from $\sim 2.7$ at $69 \mathrm{~S}$ to $\sim 3.3$ at $87 \mathrm{~S}$ ). We also found that in this frequency range the average power spectra at both stations do not exhibit any significant power enhancement. This result seems to be in dis- agreement with previous findings by Villante et al. (1997), who, in a statistical analysis of data recorded at TNB during three austral summers, found evidence for power enhancements at discrete frequencies (of the order of a few $\mathrm{mHz}$ ) and interpreted this feature as an indication for global magnetospheric modes. We think that the series of data analyzed in the present study is not long enough to provide statistical evidence for these modes, which, in the regions close to the auroral oval, can be masked by a large variety of local phenomena.

The local time dependence of the low frequency fluctu- 

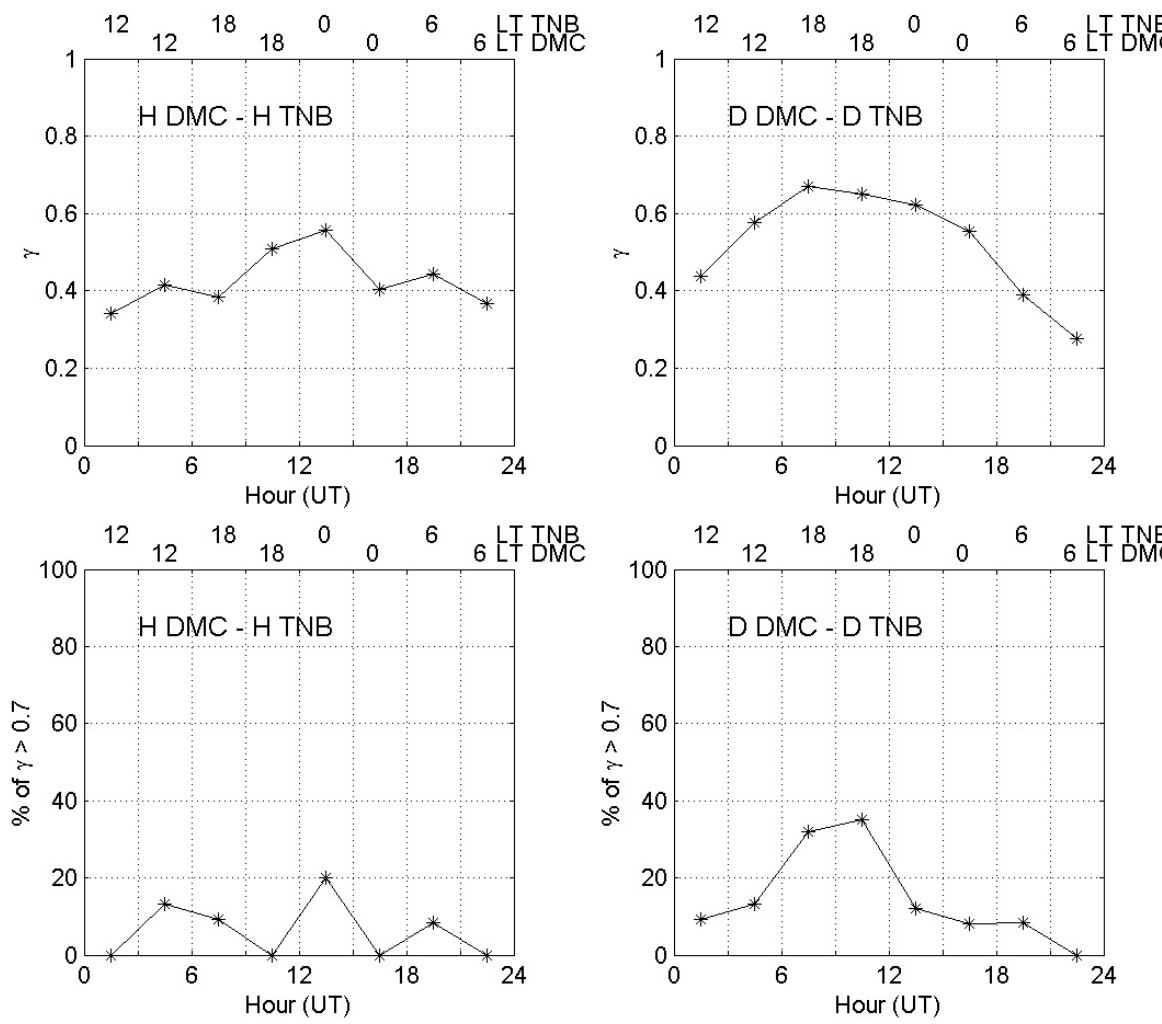

Fig. 8. Coherence $\gamma$ between the homologous components at DMC and TNB in the frequency range $0.55-1.1 \mathrm{mHz}$ : daily distribution of the average $\gamma$ values (upper panels) and of the occurrence of intervals with $\gamma>0.7$ (lower panels). Local times at the two stations are shown at the top of the plots.

ation power is different at the two stations: at TNB both components show the already known maximum around local geomagnetic noon, when the station approaches the polar cusp (Villante et al., 2000b), while at DMC the power of the $H$ and $D$ components maximizes in the local early afternoon and late morning, respectively. In order to provide an explanation for this different behaviour, it might be important to perform a similar analysis with data from stations located at intermediate latitudes between TNB and DMC. We also found that the daytime power level is definitely higher at TNB. On the contrary, during nighttime hours, when both stations are situated deep in the polar cap, the power level at DMC is somewhat higher than at TNB; this last feature is consistent with the findings by Yagova et al. (2002), who found that the nighttime low frequency power level increases with increasing latitude inside the polar cap.

At DMC the low frequency fluctuation power appears to be well related to the $K_{p}$ index and to the SW speed, with values of the correlation coefficient $\rho$ well above the $99.9 \%$ confidence level, without any clear dependence on the local time. Similar values of $\rho$ are obtained at TNB only far from the local geomagnetic noon, while around local geomagnetic noon $\rho$ exhibits a pronounced minimum (in some cases with values below the $99.9 \%$ confidence level). These results indicate that the SW speed control of the low frequency fluctuation power is stronger deep in the polar cap than close to the polar cusp. Similar findings for the relationship between Pc3-4 pulsation power at TNB and the SW speed have been recently found also by Villante et al. (2000b). In addition, in the polar cap, where the local field lines are inside the geomagnetic tail, the low frequency fluctuation power reflects well the general magnetospheric conditions, while close to the polar cusp, where the local field lines reach the most extreme magnetospheric regions, the power can also be strongly influenced by local phenomena.

The analysis of the coherence between the geomagnetic field components at the two stations shows that the fluctuations detected at the two sites are substantially decoupled for frequencies higher than $\sim 1.5 \mathrm{mHz}$, while at lower frequencies coherent fluctuations can be observed. Moreover, the coherence for the lower frequency fluctuations is statistically higher between non-homologous components, i.e. when the $H$ and $D$ components at the two stations are cross-compared; as previously remarked for the daily variation, this result could be due to the different declination at the two stations and suggests that the lower frequency geomagnetic field variations $(T=15-30 \mathrm{~min})$ could be explained mainly in terms of ionospheric phenomena related to the geographic position, rather than to field-aligned currents. We also found that the coherence is higher in the time intervals far from those in which the power level at the two stations is higher (i.e. far from the local geomagnetic noon at TNB and the local noon 

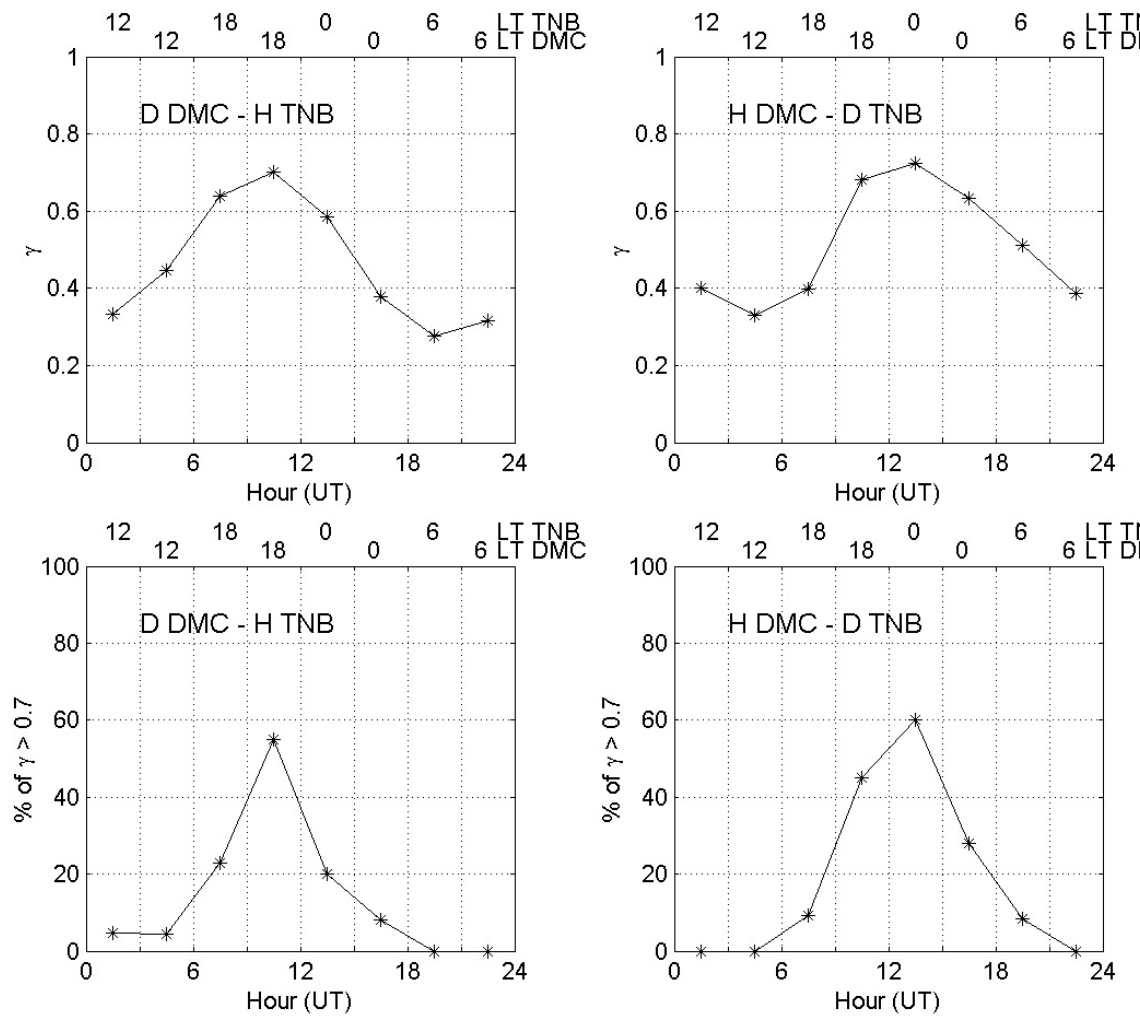

Fig. 9. The same as Fig. 8, but cross-comparing the non-homologous components at the two stations.

at DMC); in this sense it is interesting to recall the results by Yagova et al. (2002), who found that high latitude, low frequency oscillations along a magnetic meridian are highly coherent through the polar cap, while the correlation between auroral and polar cap latitudes is poor.

Acknowledgements. This research activity is supported by Italian PNRA (Programma Nazionale di Ricerche in Antartide). The campaign at DMC was supported by PNRA and IFRTP (Institut Francais pour la Recherche et la Technologie Polaires).

Topical Editor G. Chanteur thanks two referees for their help in evaluating this paper.

\section{References}

Arnoldy, R. L., Cahill, Jr., L. J., Engebretson, M. J., Lanzerotti, L. J., and Wolfe, A.: Review of hydromagnetic wave studies in the Antarctic, Rev. Geophys., 26, 181-207, 1988.

Ballatore, P., Lepidi, S., Cafarella, L., Villante, U., Meloni, A., Candidi, M., and Palangio, P.: Low frequency $(1.7-6.7 \mathrm{mHz})$ geomagnetic field fluctuations at high southern latitudes, Nuovo Cimento, 19, 517-525, 1996.

Ballatore, P., Lanzerotti, L. J., and Maclennan, C. G.: Multistation measurements of Pc5 geomagnetic power amplitudes at high latitudes, J. Geophys. Res., 103, 29 455-29 465, 1998.

Cafarella, L., Meloni, A., and Palangio, P.: Solar cycle 22 control on daily geomagnetic variation at Terra Nova Bay (Antarctica), Annali di Geofisica, 41, 805-811, 1998.
Campbell, W. H.: The regular geomagnetic field variation during quiet solar conditions, in: Geomagnetism, (Ed) Jacobs, J. A., vol. 3, 385, 1989.

Campbell, W. H.: Introduction to Geomagnetic fields, Cambridge University Press, 1997.

De Lauretis, M., Villante, U., Vellante, M., and Wolfe, A.: An analysis of power spectral indices in the micropulsation frequency range at different ground stations, Planet. Space Sci., 39, 975982, 1991.

Detrick, D. L. and Lanzerotti, L. J.: Geomagnetic quiet time $(\mathrm{Sq})$ variations at high latitudes, Geophys. Res. Lett., 28, 2581-2584, 2001.

Engebretson, M. J., Hughes, W. J., Alford, J. L., Zesta, E., Cahill Jr., L. J., Arnoldy, R. L., and Reeves, G. D.: Magnetometer array for cusp and cleft studies observations of the spatial extent of broadband ULF magnetic pulsations at cusp/cleft latitudes, J. Geophys. Res., 100, 19371-19386, 1995.

Engebretson, M. J., Glassmaier, K. H., and Stellmark, M.: The dependence of high latitude Pc5 wave power on solar wind velocity and on the phase of high speed solar wind streams, J. Geophys. Res., 103, 26 271-26 283, 1998.

Kleimenova, N. G., Kosyreva, O. V., Bitterly, J., and Folk, J.: Spectrum of long-period geomagnetic pulsations $(n p)$ in the region of the daytime polar cusp, Geomagn. Aeron., 29, 742-743, 1989.

Kurazhkovskaya, N. A. and Klain, B. I.: Peculiar features of burst series of long period irregular ipcl-type geomagnetic pulsations in the vicinity of the dayside cusp, Intern. J. Geomagn. Aeron., 2,2000

Lepidi, S., Francia, P., Villante, U., Lanzerotti, L. J., and Meloni, A.: Polarization pattern of low frequency geomagnetic field 
fluctuations $(0.8-3.6 \mathrm{mHz})$ at high and low latitude, J. Geophys. Res., 104, 305-310, 1999.

Matthews, D. L., Ruohoniemi, J. M., Dudeney, J. R., Farrugia, C. F., Lanzerotti, L. J., and Friis-Christensen, E.: Conjugate cuspregion ULF pulsation responses to the solar wind event of 23 May 1989, J. Geophys. Res., 101, 7829-7842, 1996.

Nishida, T. and Kokubun, S.: New polar magnetic disturbance: Sqp, SP, DCP and Dp2, Rev. Geophys. Space Phys., 9, 417-425, 1971.

Olson, J. V.: ULF signatures of the Polar cusp, J. Geophys. Res., 91, 10 055-10062, 1986.

Palangio, P., Meloni, A., Cafarella, L., and Zirizzotti, A.: Magnetic field measurements at Terra Nova Bay geomagnetic observatory, in: Italian Geophysical Observatories in Antarctica, (Eds) Meloni, A. and Morelli, A., 21, 1996.

Samson, J. C., Harrold, B. G., Ruohoniemi, J. M., Greenwald, R. A., and Walker, A. D. M.: Field line resonances associated with MHD waveguides in the magnetosphere, Geophys. Res. Lett., 19, 441-448, 1992.

Troitskaya, V. A., Bolshakova, O. V., and Hessler, V. B.: Irregular geomagnetic pulsations in the polar cap, Rep. Assem. IAGA, Int. Assoc. Geom. and Aeron., Rockville, Md., 1973.

Villante, U., Lepidi, S., Francia, P., Meloni, A., and Palangio, P.: Long period geomagnetic field fluctuations at Terra Nova Bay (Antartica), Geophys. Res. Lett., 24, 1443-1446, 1997.

Villante, U., Lepidi, S., Francia, P., Vellante, M., Meloni, A., and
Palangio, P.: ULF fluctuations at Terra Nova Bay (Antartica), Annali di Geofisica, 43, 217-227, 2000a.

Villante, U., Vellante, M., and De Sanctis, G.: An analysis of Pc3 and Pc4 pulsations at Terra Nova Bay (Antarctica), Ann. Geophysicae, 18, 1412-1421, 2000b.

Walker, A. D. M., Ruohoniemi, J. M., Baker, K. B., Greenwald, R. A., and Samson, J. C.: Spatial and temporal behaviour of ULF pulsations observed by the Goose Bay HF radar, J. Geophys. Res, 97, 12 187-12 202, 1992.

Wolfe, A., Kamen, E., Lanzerotti, L. J., Maclennan, C. G., Bamber, J. F., and Venkatesan, D.: ULF geomagnetic power at cusp latitudes in response to upstream solar wind conditions, J. Geophys. Res, 92, 168-174, 1987.

Yagova, N. V., Lanzerotti, L. J., Villante, U., Pilipenko, V. A., Lepidi, S., Francia, P., Papitashvili, V. O., and Rodger, A. S.: ULF Pc5-6 magnetic activity in the polar cap as observed along a geomagnetic meridian in Antartica, J. Geophys. Res, 107, SMP 221-SMP22-12, 2002.

Ziesolleck, C. W. S. and McDiarmid, D. R.: Auroral latitude Pc5 field line resonances: quantized frequencies, spatial characteristics and diurnal variations, J. Geophys. Res., 99, 5817-5830, 1994.

Ziesolleck, C. W. S. and McDiarmid, D. R.: Statistical survey of auroral latitude Pc5 spectral and polarization characteristics, J. Geophys. Res., 100, 19299-19312, 1995. 